Louisiana State University

LSU Digital Commons

Faculty Publications

Department of Chemistry

$10-1-2017$

\title{
The effect of a crosslinking chemical reaction on pattern formation in viscous fingering of miscible fluids in a Hele-Shaw \\ cell
}

\author{
Patrick H. Bunton \\ William Jewell College \\ Michael P. Tullier \\ Louisiana State University \\ Eckart Meiburg \\ University of California, Santa Barbara \\ John A. Pojman \\ Louisiana State University
}

Follow this and additional works at: https://digitalcommons.Isu.edu/chemistry_pubs

\section{Recommended Citation}

Bunton, P., Tullier, M., Meiburg, E., \& Pojman, J. (2017). The effect of a crosslinking chemical reaction on pattern formation in viscous fingering of miscible fluids in a Hele-Shaw cell. Chaos, 27 (10)

https://doi.org/10.1063/1.5001285

This Article is brought to you for free and open access by the Department of Chemistry at LSU Digital Commons. It has been accepted for inclusion in Faculty Publications by an authorized administrator of LSU Digital Commons.

For more information, please contact ir@lsu.edu. 


\section{UC Santa Barbara}

UC Santa Barbara Previously Published Works

\section{Title}

The effect of a crosslinking chemical reaction on pattern formation in viscous fingering of miscible fluids in a Hele-Shaw cell.

\section{Permalink}

https://escholarship.org/uc/item/7s67w5x1

Journal

Chaos (Woodbury, N.Y.), 27(10)

\section{ISSN}

1054-1500

\section{Authors}

Bunton, Patrick $\mathrm{H}$

Tullier, Michael $\mathrm{P}$

Meiburg, Eckart

et al.

\section{Publication Date}

2017-10-01

\section{DOI}

10.1063/1.5001285

Peer reviewed 
The effect of a crosslinking chemical reaction on pattern formation in viscous fingering of miscible fluids in a Hele-Shaw cell

Patrick H. Bunton, Michael P. Tullier, Eckart Meiburg, and John A. Pojman

Citation: Chaos 27, 104614 (2017); doi: 10.1063/1.5001285

View online: http://dx.doi.org/10.1063/1.5001285

View Table of Contents: http://aip.scitation.org/toc/cha/27/10

Published by the American Institute of Physics 


\title{
The effect of a crosslinking chemical reaction on pattern formation in viscous fingering of miscible fluids in a Hele-Shaw cell
}

\author{
Patrick H. Bunton, ${ }^{1}$ Michael P. Tullier, ${ }^{2}$ Eckart Meiburg, ${ }^{3, a)}$ and John A. Pojman ${ }^{2}$ \\ ${ }^{1}$ Department of Physics and Mathematics, William Jewell College, Liberty, Missouri 64068, USA \\ ${ }^{2}$ Department of Chemistry, Louisiana State University, Baton Rouge, Louisiana 70803, USA \\ ${ }^{3}$ Department of Mechanical Engineering, University of California at Santa Barbara, Santa Barbara, \\ California 93106, USA
}

(Received 26 April 2017; accepted 21 July 2017; published online 18 September 2017)

\begin{abstract}
Viscous fingering can occur in fluid motion whenever a high mobility fluid displaces a low mobility fluid in a Darcy type flow. When the mobility difference is primarily attributable to viscosity (e.g., flow between the two horizontal plates of a Hele-Shaw cell), viscous fingering (VF) occurs, which is sometimes termed the Saffman-Taylor instability. Alternatively, in the presence of differences in density in a gravity field, buoyancy-driven convection can occur. These instabilities have been studied for decades, in part because of their many applications in pollutant dispersal, ocean currents, enhanced petroleum recovery, and so on. More recent interest has emerged regarding the effects of chemical reactions on fingering instabilities. As chemical reactions change the key flow parameters (densities, viscosities, and concentrations), they may have either a destabilizing or stabilizing effect on the flow. Hence, new flow patterns can emerge; moreover, one can then hope to gain some control over flow instabilities through reaction rates, flow rates, and reaction products. We report effects of chemical reactions on VF in a Hele-Shaw cell for a reactive step-growth cross-linking polymerization system. The cross-linked reaction product results in a non-monotonic viscosity profile at the interface, which affects flow stability. Furthermore, threedimensional internal flows influence the long-term pattern that results. Published by AIP Publishing. [http://dx.doi.org/10.1063/1.5001285]
\end{abstract}

Viscous fingering is a phenomenon observed when a more viscous fluid displaces a less viscous fluid, often in a porous medium. The resultant viscosity gradients create fingershaped features at the fluid interface. A rich variety of patterns can be observed if a chemical reaction alters the viscosity at the interface. By varying the concentration of initiator in a polymerization reaction at the interface between displaced and displacing monomer fluids in a Hele-Shaw cell, it was possible to have some measure of control over the viscosity change. Schlieren imaging was used to observe the resulting fingering patterns.

\section{INTRODUCTION}

Ilya Prigogine often spoke in his lectures at the University of Texas, when one of us (JAP) was a graduate student, about the emergence of patterns far from equilibrium, citing pattern formation in Rayleigh-Bénard convection as an archetypal example of nonequilibrium self-organization. Another type of self-organizing system with fluids is viscous fingering (VF) in which a fluid of lower viscosity is forced into one of higher viscosity, resulting in complex patterns that can be modulated by chemical reactions. ${ }^{1-6}$ In this work, we report our experimental study on VF in a Hele-Shaw cell with miscible fluids, and how a crosslinking chemical reaction at the flow front modulates the flow pattern.

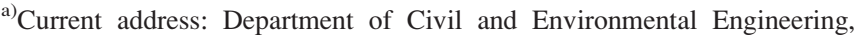
Stanford University, Stanford, CA 94305, USA.
}

During the past few decades, there have been extensive experimental and computational studies of fingering and related flow instabilities in Hele-Shaw cells. ${ }^{7-11}$ A Hele-Shaw cell is simply two flat plates, often glass, separated by a narrow gap. Generally the gap is filled with one fluid of certain properties that is then displaced by another fluid with carefully selected properties such as density, viscosity, species concentration, particle loading, miscibility, interfacial tension, reactivity, $\mathrm{pH}$, or temperature. Hele-Shaw cells have been used extensively because of the comparative ease of imaging the flows along with the significant computational simplification of dealing with a nominally two-dimensional system. In addition, flow through a narrow gap is mathematically analogous to the significant engineering, geophysical, and environmental issues associated with flow through porous media via a simple scaling of permeability to the square of the gap. ${ }^{7}$

Fingering can occur any time a more mobile fluid displaces a less mobile fluid in a Hele-Shaw cell or porous medium. For flows in a gravity field such as vertical Hele-Shaw cells or layering of differing temperatures or salt concentrations in the ocean, a number of instabilities can develop. Primarily driven by gravity and density difference, these include the Rayleigh-Taylor instability, doublediffusive fingering, diffusive-layer convection, and other more elaborate instabilities such as staircases and leaking modes in sediment-laden flows. ${ }^{12}$ Herein, we discuss viscous fingering where the difference in mobility is primarily the result of differing viscosities. This case is generally studied in horizontal Hele-Shaw cells in an attempt to isolate the 
viscous effects from the gravitational effects. Nonetheless, in the work reported herein, gravitational effects modulate these horizontal viscous flows altering the long-term pattern formed.

Horizontal Hele-Shaw flows are divided into two broad classes, depending on whether the flows are immiscible or miscible. Immiscible flows are characterized by the capillary number $\mathrm{Ca},{ }^{7}$ which compares the viscous force to the surface tension force. In miscible flows the effect of interfacial tension is widely regarded to be absent, though this has been called into question in some recent studies. ${ }^{13-19}$ Miscible flows are characterized by the Péclet number, which compares rate of advection to diffusion. ${ }^{20}$ Flows can also be characterized as to whether the fluids involved are Newtonian (constant viscosity) or non-Newtonian (viscosity varies with shear rate) with widely differing outcomes. In particular, shear thinning can lead to very narrow fingers reminiscent of diffusion-limited aggregation. ${ }^{21}$ This can be particularly relevant to liquid polymers or aqueous polymer solutions, which one should not confuse with hydrodynamics wherein the polymerization reaction is actually occurring during the flow as is discussed herein.

That the occurrence of chemical reactions at the interface potentially alters the hydrodynamics for any of the scenarios above as has been clearly and concisely summarized recently by De Wit. ${ }^{22}$ Reactions generally alter the density and viscosity at or possibly trailing behind the interface (depending on the reaction rate), potentially producing an entirely new fluid, gel, or precipitate there. Along with the Péclet number, reactive flows are further characterized by the Damköhler number (Da), which compares the reaction rate to the advection rate, potentially further scaled to mass diffusivity. Linear stability analyses have indicated that a non-monotonic viscosity profile at the interface can stabilize otherwise unstable viscous flows. ${ }^{2,23}$ In many cases, Da is large and experimentalists have little if any control over the rate of reaction. Polymerization reactions can provide a venue where one can control the reaction rate via the concentration of the initiator or catalyst, which is what is done herein.

Since reactive hydrodynamic instabilities in Hele-Shaw cells have been summarized by De Wit, ${ }^{22}$ we here highlight only a carefully selected subset of the directly applicable related literature. Nagatsu et al. varied the viscosity at the interface of an aqueous polymer solution and aqueous $\mathrm{HCl}$ for a diffusion-limited reaction. Injecting a less-viscous $\mathrm{NaOH}$ solution into a more viscous 0.5 wt. \% poly(acrylic acid) (PAA) solution resulted in an increase in viscosity whereas a viscosity decrease occurred when $\mathrm{HCl}$ solution was injected into 0.5 wt. \% PAA solution including $0.065 \mathrm{~mol} / \mathrm{l} \mathrm{NaOH}$ as a consequence of the $\mathrm{pH}$ dependence of the polymer size. ${ }^{24}$ The authors characterized the flows primarily in terms of the area occupied by the fingers (possibly suggestive of more or less efficient displacement as applied to petroleum recovery). They found that for reactions that increased the viscosity, the occupied area was larger than for the non-reactive system. In contrast, reactions that decreased the viscosity resulted in a smaller area occupied by the fingers. They carefully accounted for the possibility of shear thinning and viscoelastic effects and operated in a regime of shear rate and concentration where there were no elastic components. Nagatsu et al. studied another polymer system which under certain conditions produced a spiral pattern rather than the normal fingering in a Hele-Shaw cell. ${ }^{25}$ The displacing fluid was a trivalent iron solution of $\mathrm{Fe}\left(\mathrm{NO}_{3}\right)_{3}$ and the displaced fluid was aqueous pol$\mathrm{y}$ (sodium acrylate) (SPA). They attribute the spiral pattern to the formation of a gel at the interface of the iron solution and the SPA. Riolfo et al. demonstrated computationally that a chemical reaction can destabilize an otherwise stable interface via a nonmonotonic viscosity profile formed at the interface by either increasing or decreasing the viscosity at the interface. They further demonstrated this effect experimentally using the $\mathrm{pH}$ dependence of viscosity of certain polymer solutions. ${ }^{26}$ Podgorski et al. performed an intriguing study of the several flow patterns that result in a Hele-Shaw cell when two fluids react that form a gel-like membrane at their interface. ${ }^{27}$ In particular, they used a cationic surfactant and an ionic salt, which form a "wormlike micellar fluid" when mixed. They observed a number of flow patterns including "mushrooms," a "fanlike" pattern with a flowerlike appearance that transitioned to "tentacles," a regular pattern of fans of well-defined wavelength, as well as tentacles without fans. All of these patterns were explained phenomenologically from gel formation and occasional gel rupturing at the interface. They contrast the gels so formed with those of typical polymer fluids since the constituents were in a dynamic equilibrium with solution due to breaking and reforming. ${ }^{27}$

Moving to the case at hand, an example of a twocomponent system of miscible fluids where a viscosity change can occur at the interface where the two fluids mix is a polymerization reaction between two liquid monomers. The change from small individual monomer molecules to large polymers causes an increase in viscosity due to the entanglement of the polymer chains. In a step-growth polymerization, two separate chemical groups must come together to participate in the polymer forming reaction. If two monofunctional components are used, the coupling reaction will yield a dimer with little to no viscosity increase (see Fig. 1). If two difunctional components are used, a linear polymer will form with an alternating structure, which leads to an increase in viscosity. If the functionality of one of the components is further increased to three or more, the polymer system will form a continuous network and lead to a dramatic viscosity increase or even to a solid. These reactions at or behind the front not only affect the nature of the flow, but also the character and pattern of the output product.

In addition to the functionality of the monomers, the rate of the polymerization reaction is another way to control the viscosity at the interface between the two monomers. For a given system, the more rapid the polymerization, the faster the viscosity will increase. The reaction rate for step-growth polymerizations can be controlled through the monomer concentration or the initiator concentration, if one is required for the reaction. Since the greatest viscosity change will result from polymer systems with no solvent, the initiator concentration is the more practical parameter to modulate. The initiator can be dissolved into one of the two components where it remains unreactive until the other monomer is introduced into the system. In this way, the polymerization reaction and 


\section{I. $\mathrm{A}+\mathrm{B} \rightarrow \mathrm{A}-\mathrm{B}$

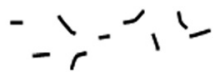

II. $\mathrm{A}-\mathrm{A}+\mathrm{B}-\mathrm{B} \rightarrow-\mathrm{A}-\mathrm{A}-\mathrm{B}-\mathrm{B}-$

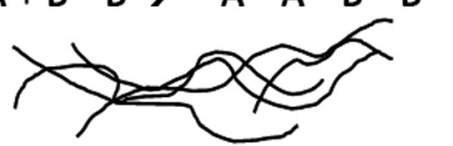

III. $A-E_{A}^{A}+B-B \rightarrow-B-B-A-C_{A-B-B}^{A-B-B}-$

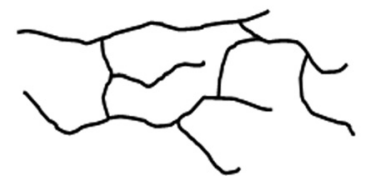

FIG. 1. The effect of monomer functionality of thiol-acrylate coupling: I. Monofunctional monomers give small molecules with little increase in viscosity. II. Difunctional monomers produce linear polymer molecules that form liquids whose viscosities depend on the length of the molecules. III. Having at least one trifunctional monomer gives an interconnected network with a significant viscosity increase.

subsequent increase in viscosity can be limited to the interface between the two components.

Thiol-acrylate polymerizations are examples of stepgrowth reactions that require an initiator or catalyst to proceed. The Michael type addition of a thiol to an acrylate (see Fig. 2) is base catalyzed and was first reported in $1947 . .^{28,29}$ There are a wide variety of commercially available thiols and acrylates, so selecting a system with the desired functionalities and initial viscosities is relatively straightforward. These reactions proceed rapidly at room temperature, can be run without solvent, and use non-hazardous materials. While the reaction requires a chemical base to proceed, several initiation strategies are possible but will not be discussed here. ${ }^{30}$ In each case though, the higher the catalyst/initiator concentration, the faster the reaction will proceed. Applications of thiol-acrylate chemistry range from coupling reactions between monofunctional species ${ }^{31-33}$ to the fabrication of microparticles, ${ }^{34}$ polymer materials for microfluidics, ${ }^{35,36}$ biocompatible tissue scaffolds, ${ }^{37,38}$ hydrogels, ${ }^{39,40}$ and polymers with a variety of architectures. $^{41-44}$

\section{EXPERIMENTAL}

Schlieren imaging was used to monitor viscous fingering during step-growth polymerization in a horizontal Hele-Shaw cell. Primary lenses were $15 \mathrm{~cm}$ telescope objective lenses, which yielded an overall field of view of $13 \mathrm{~cm}$ in diameter. The Hele-Shaw cell consisted of two $6 \mathrm{~mm}$ thick optical quality glass plates separated by gaskets of precision silicone held

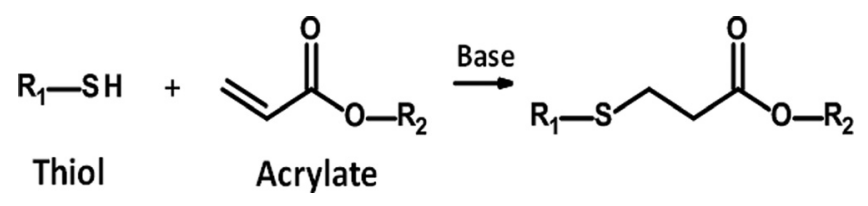

FIG. 2. General base-catalyzed thiol-acrylate coupling reaction (R represents any carbon-based group). down by their own weight or with added weights. The typical flow rate was $0.2 \mathrm{ml} / \mathrm{min}$ with a gap of $0.35 \mathrm{~mm}$. Images were collected using either a Point Grey machine vision camera or a Nikon camera and telephoto lens. The technique has been described in more detail elsewhere. ${ }^{45}$ A solution of a $2,2^{\prime}$-(ethylenedioxy)-diethanethiol (dithiol or DT), a difunctional thiol, containing varying concentrations of octylamine (OA) as initiator was used to displace trimethylolpropane triacrylate (TMPTA), a trifunctional acrylate, using a syringe pump. OA concentration was measured in parts per hundred thiol (PPTh) by mass (e.g., $1 \mathrm{~g}$ OA mixed with $100 \mathrm{~g}$ DT gives a 1 PPTh mixture).

The concentration of the initiator (OA) was used to control the rate of reaction between the two components (thiol and acrylate). Despite the horizontal orientation of the cell and the small gap, evidence of a Rayleigh-Taylor instability was observed, which seemed to have significant effects on the long-term resultant flow patterns.

\section{RESULTS AND DISCUSSION}

Dithiol (DT), containing different concentrations of the initiator octylamine (OA), displaced TMPTA in a horizontal Hele-Shaw cell. In each case, the flow rate was $0.2 \mathrm{ml} / \mathrm{min}$ into a $0.35 \mathrm{~mm}$ gap. The viscosity of DT was measured in house using a Brookfield viscometer with small sample adapter to be $5 \mathrm{mPa} \cdot \mathrm{s}$ at $20^{\circ} \mathrm{C}$ while that of TMPTA was reported by Allnex as a range from $80-135 \mathrm{mPa} \cdot \mathrm{s}$ at $25^{\circ} \mathrm{C}$; hence, the displaced fluid is significantly more viscous than the displacing. The reaction rate increases with increasing concentration of OA resulting in a more viscous fluid, a gel, or even a solid product. We were unable to measure the viscosity of the reacted polymer without significant risk to the rheometer; however, our measured viscosities of another dithiol reacted with a di-functional acrylate were in the 1000 to $12000 \mathrm{mPa}$ s range, albeit with a different initiator and over a longer time scale. The viscosities of di-functional acrylates with triacrylates should be significantly higher still due to the ability to crosslink. Indeed, at high reaction rates, the output product eventually became a solid. Since Schlieren imaging is sensitive to gradient in the index of refraction, and given that one expects polymerization to increase density and consequently the index of refraction, the darker regions in the figures below likely correspond to greater conversion and/or cross-linking which was further confirmed visually by the reaction product remaining on the plates. Quite varied patterns emerged as the reaction rate was increased, as shown in Fig. 3. Without any reaction, we have the classical case of a less viscous fluid displacing a more viscous one, which results in the well-known viscous fingering instability [Fig. 3(a)] that gives rise to such mechanisms as shielding, merging and splitting. ${ }^{1}$ For small and moderate concentrations of OA (frames b-d), the overall shape of the displacement front remains qualitatively similar; however, narrow streamwise structures emerge that are superimposed on the wider primary fingers. These can be seen especially clearly in the 0.3 PPTh OA flow, but are apparent at lower reaction rates upon close examination. Similar structures have previously been observed in non- 

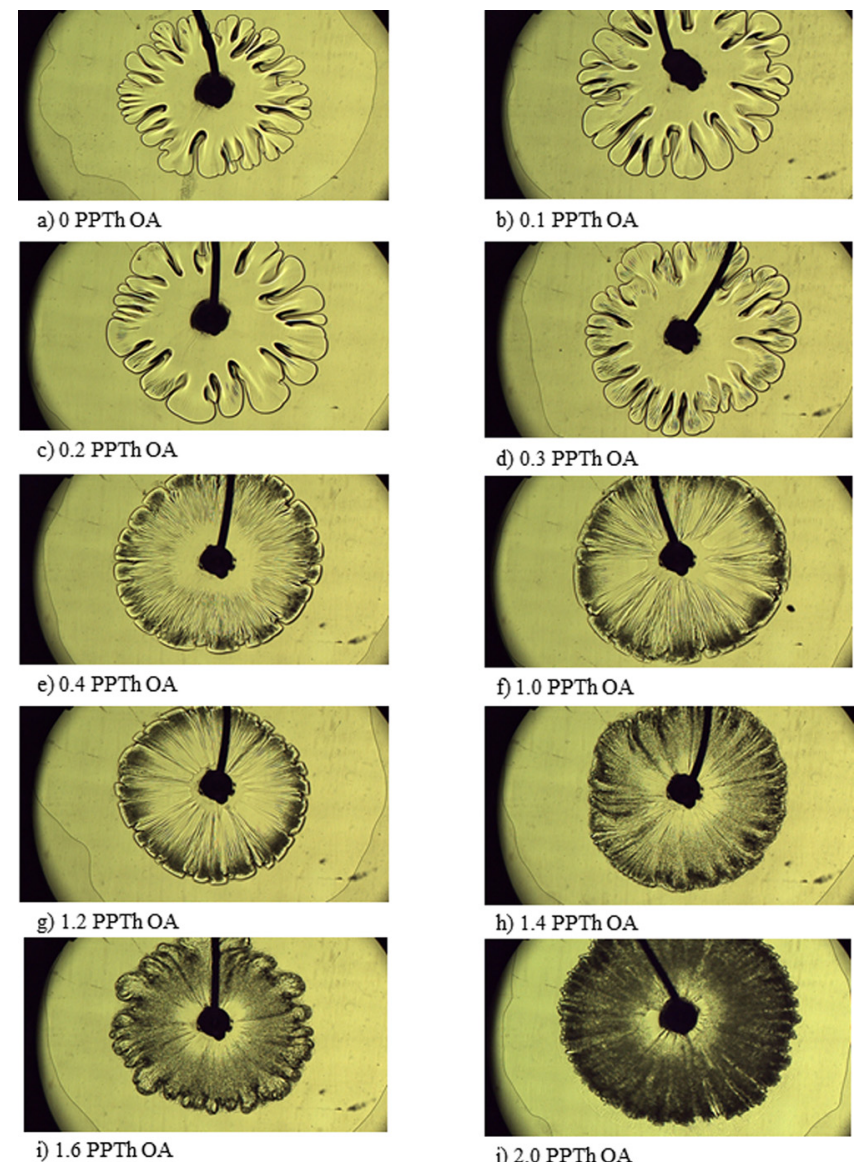

j) $2.0 \mathrm{PPThOA}$

compares the resultant pattern for initiator concentrations of 0 PPTh, 0.3 PPTh, and 0.4 PPTh.

The density of the dithiol was reported by the manufacturer to be $1.12 \mathrm{~g} / \mathrm{cm}^{3}$ while that of the TMPTA was measured in house as $1.106 \mathrm{~g} / \mathrm{cm}^{3}$ at $28^{\circ} \mathrm{C}$. The assumed Poiseuille flow is gravitationally unstable regardless of whether the density increases or decreases, with the distinction merely being whether the upper or lower interface is the unstable one. While the density can increase because of the polymerization reaction, this reaction is exothermic, which can decease the density. However, the increase in density from polymerization should dominate over temperature effects. In particular, the $0.35 \mathrm{~mm}$ gap between two approximately $6 \mathrm{~mm}$ thick glass plates limits the temperature rise. We do note that Taylor dispersion effects may further complicate this Poiseuille profile interpretation.

For OA concentrations of 0.4 PPTh or larger, we notice that the leading edge of the front tends to approach a roughly circular shape indicative of a stable displacement. This can be explained in the following way: at these higher OA concentrations, the chemical reaction along the interface produces sufficiently large amounts of polymer to result in a substantial increase of the local viscosity in the interfacial region. The viscosities of the reaction products are expected to be an order of magnitude or more higher than the reactants as was discussed earlier based on measurements of difunctional thiol-acrylates. Hence, eventually the viscosity in the interfacial region will exceed that of the displaced fluid, so that the circumferentially averaged viscosity profile becomes non-monotonic, with a maximum in the interfacial region. That the profile is non-monotonic follows from the fact that the fluid behind the front is still emerging unreacted, but that evidence of a reaction product appears at the front in the form of the dark regions in the images. Furthermore, cleaning the Hele-Shaw plates after the experiment clearly revealed a gel or solid for higher reaction rates. The effects of such non-monotonic viscosity profiles on displacement processes have been analyzed theoretically and computationally in the past. ${ }^{2,3,47,48}$

These investigations seem consistent with the displacement being locally unstable in those regions where the mobility profile is unfavorable and locally stable where it is favorable. Such behavior is noticeable for OA concentrations in the range of 0.4 to 1.2 PPTh. The term "unfavorable" for conditions commensurate with VF comes from the petroleum industry where less viscous fluids, such as water, are injected into one well with the goal of forcing petroleum out of a neighboring production wells. This recovery and related techniques are termed "secondary recovery" or "enhanced
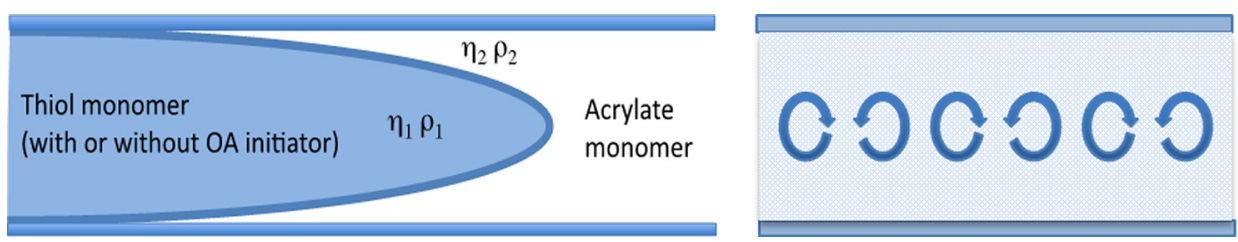

FIG. 4. The Poiseuille profile of the flow leads to a gravitational instability of a more dense fluid overlying a less dense fluid regardless of whether the displacing or the displaced fluid is denser. Left: flow viewed from the side. Right: flow viewed coming toward you. Left figure adapted from Haudin $e t$ al. ${ }^{46}$ 

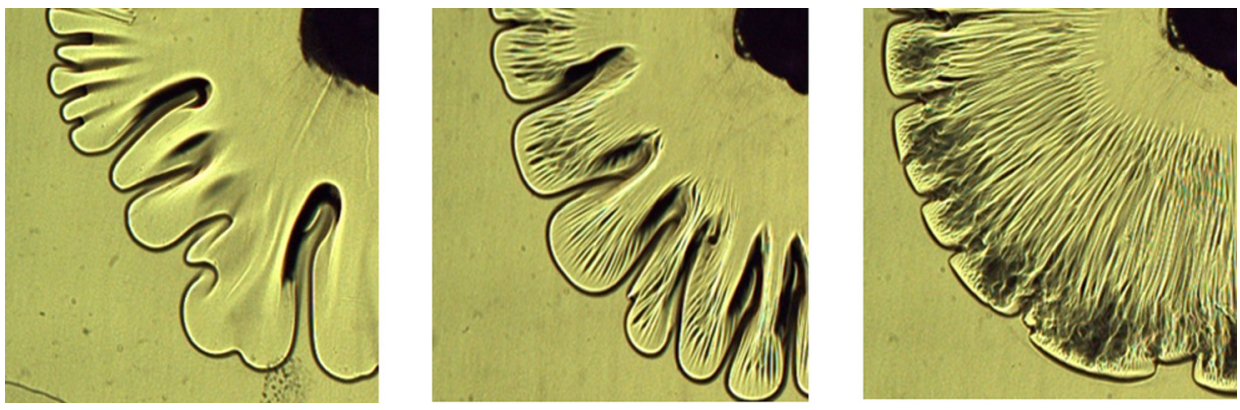

FIG. 5. Left: no reaction. Center: 0.3 PPTh OA initiator. Right: 0.4 PPTh OA initiator. As the reaction rate increases, three-dimensional effects begin first to show up and then dominate the flow pattern. The width of the images is approximately $5 \mathrm{~cm}$. The flow rate was $0.2 \mathrm{ml} / \mathrm{min}$ into a $0.35 \mathrm{~mm}$ gap. recovery. ${ }^{, 49}$ Fingering of the less viscous fluid through the underground porous medium decreases the sweep efficiency and extraction of the petroleum, which may essentially vanish altogether once "breakthrough" occurs. Breakthrough refers to when one or more fingers penetrate completely though the fluid that is being displaced (in this case petroleum) and thereafter bypass it. The term "favorable" in this context of course refers to the stable case leading to efficient sweeping of hydrocarbons from the reservoir.

For even larger OA concentrations, the frontal dynamics appear to become even more complex. Here the reaction products may no longer be Newtonian, and viscoelastic effects may gain importance. At sufficiently high reaction rates, the product may take the form of a gel and eventually a solid, with implications for the stability of the displacement front. For example, for an OA concentration of 1.6 PPTh, we notice that the leading edge of the front no longer has an approximately circular shape, and instead finger-like structures appear once again, indicating an overall unstable nature of the displacement. These structures may be similar to the mushroom and breakout mechanism proposed by Podgorski et $a .^{27} \mathrm{~A}$ detailed understanding of the mechanisms driving these structures will require additional experimental and theoretical efforts. Clearly, the overall dynamics of the present reactive flow system is governed by a complex and intricate balance of several competing mechanisms. In regions of vigorous fingering or buoyancy-driven instability, the interface deforms strongly, so that additional interfacial area is rapidly generated, which will enhance the rate at which reaction product forms, with implications for the local viscosity. In turn, this will affect the further growth of the instability. On the other hand, in more stable regions, the rate at which interfacial area is generated is lower, so that a less reaction product forms.

We also note that in the present study we did not attempt to analyze the dependence of the displacement front dynamics on the injection rate. It will be interesting to investigate the influence of the ratio of the reactive and convective time scales, which give rise to a Damköhler number. In particular, one can envision cases where the reactions keep pace with the front as well as cases where it lags behind. This is complicated by what one even means by the "front." From a twodimensional standpoint, the front would be the outermost boundary of the fingers. However, for miscible Poiseuille flow, the reaction is occurring at or behind the Poiseuille interface. Clearly, the degree or extent to which a nonmonotonic profile can occur depends on the rate at which the viscosity is increasing as compared to the rate at which the front passes by.

\section{CONCLUSION}

Thiol-acrylate miscible polymer systems exhibit a variety of flow patterns as the reactivity is increased. Surprisingly, buoyancy-driven convection in the form of Rayleigh-Taylor instability that leads to counter-rotating vortices in the direction of the displacement seems to play a significant role in the flows for reactive situations but not for unreactive ones. These vortices imprint their presence on the long-term pattern of the output product when the reaction rate is sufficient for the reaction product to be a gel for the cross-linking system studied herein. At moderate to high reaction rates, the system is highly nonlinear with the flow structure and output product depending on the interplay of flow rate, reaction rate, exothermicity, diffusion rate, and functionality of the monomers. These complex, nonlinear behaviors, which strongly affect long-term pattern formation, are exactly the type of system for which Prigogine provided the theoretical underpinning that helped to make them legitimate objects of study.

\section{ACKNOWLEDGMENTS}

We acknowledge support from the National Science Foundation (CBET-1335739, CBET-1511653, and CBET1438052).

${ }^{1}$ P. G. Saffman, J. Fluid Mech. 173, 73-94 (1986).

${ }^{2}$ O. Manickam and G. M. Homsy, Phys. Fluids A: Fluid Dyn. 5(6), 1356-1367 (1993).

${ }^{3}$ O. Manickam and G. M. Homsy, Phys. Fluids 6(1), 95-107 (1994).

${ }^{4}$ A. De Wit and G. M. Homsy, Phys. Fluids 11, 949-951 (1999).

${ }^{5}$ A. De Wit and G. M. Homsy, J. Chem. Phys. 110, 8663-8675 (1999).

${ }^{6}$ R. Demuth and E. Meiburg, Phys. Fluids 15, 597 (2003).

${ }^{7}$ G. M. Homsy, Annu. Rev. Fluid Mech. 19, 271-311 (1987).

${ }^{8}$ F. Heussler, R. Oliveira, M. John, and E. Meiburg, J. Fluid Mech. 752, 157-183 (2014).

${ }^{9}$ M. O. John, R. M. Oliveira, F. H. C. Heussler, and E. Meiburg, J. Fluid Mech. 721, 295-323 (2013).

${ }^{10}$ R. M. Oliveira and E. Meiburg, J. Fluid Mech. 687, 431-460 (2011).

${ }^{11}$ A. Aubertin, G. Gauthier, J. Martin, D. Salin, and L. Talon, Phys. Fluids 21, 054107 (2009).

${ }^{12}$ P. G. Drazin and W. H. Reid, Hydrodynamcs Stability (Cambridge University Press, 1981).

${ }^{13}$ S. Pramanik and M. Mishra, Phys. Rev. E 91, 03300 (2015).

${ }^{14} \mathrm{C}$. Chen and Y. Liu, Int. J. Dyn. Fluids 1, 55-66 (2005).

${ }^{15}$ C. Chen, C. W. Huang, H. Gadelha, and J. A. Miranda, Phys. Rev. E 78, 016306 (2008).

${ }^{16}$ R. Borcia and M. Bestehorn, Langmuir 25, 1919-1922 (2009).

${ }^{17}$ T. J. Hughes, A. Honori, B. F. Graham, A. S. Chauhan, M. L. Johns, and E. F. May, Int. J. Greenhouse Gas Control 9, 457-468 (2012).

${ }^{18}$ S. Pramanik and M. Mishra, Chem. Eng. Sci. 110, 144-152 (2014). 
${ }^{19}$ T. H. Beeson-Jones and A. W. Woods, J. Fluid Mech. 782, 127-143 (2015).

${ }^{20}$ L. G. Leal, Advanced Transport Phenomena: Fluid Mechanics and Convective Transport Process (Cambridge University Press, 2010).

${ }^{21}$ A. Lindner, P. Coussot, and D. Bonn, Phys. Rev. Lett. 85(2), 314 (2000).

${ }^{22}$ A. De Wit, Philos. Trans. A 374, 20150419 (2016).

${ }^{23}$ D. Schafroth, N. Goyal, and E. Meiburg, Eur. J. Mech. B: Fluids 26, 444 (2007).

${ }^{24}$ Y. Nagatsu, K. Matsuda, Y. Kato, and Y. Tada, J. Fluid Mech. 571, 475-493 (2007).

${ }^{25}$ Y. Nagatsu, A. Hayashi, M. Ban, Y. Kato, and Y. Tada, Phys. Rev. E 78, 026307 (2008).

${ }^{26}$ L. A. Riolfo, Y. Nagatsu, S. Iwata, R. Maes, P. M. J. Trevelyan, and A. De Wit, Phys. Rev. E 85, 015304 (2012).

${ }^{27}$ T. Podgorski, M. C. Sostarecz, S. Zorman, and A. Belmonte, Phys. Rev. E 76, 016202 (2007).

${ }^{28}$ L. L. Gershbein and C. D. Hurd, J. Am. Chem. Soc. 69(2), 241-242 (1947).

${ }^{29}$ C. D. Hurd and L. L. Gershbein, J. Am. Chem. Soc. 69(10), 2328-2335 (1947).

${ }^{30}$ J. W. Chan, C. E. Hoyle, A. B. Lowe, and M. Bowman, Macromolecules 43, 6381-6388 (2010).

${ }^{31}$ D. P. Nair, M. Podgórski, S. Chatani, T. Gong, W. Xi, C. R. Fenoli, and C. N. Bowman, Chem. Mater. 26(1), 724-744 (2014).

${ }^{32}$ M. W. Jones, G. Mantovani, S. M. Ryan, X. Wang, D. J. Brayden, and D. M. Haddleton, Chem. Commun. O(35), 5272-5274 (2009).

${ }^{33}$ S. E. R. Auty, O. Andren, M. Malkoch, and S. P. Rannard, Chem. Commun. 50(50), 6574-6577 (2014).

${ }^{34}$ C. O. Bounds, R. Goetter, J. A. Pojman, and M. Vandersall, J. Polym. Sci. A: Polym. Chem. 50, 409-422 (2012).

${ }^{35}$ W. Zhang, M. P. Tullier, K. Patel, A. Carranza, J. A. Pojman, and A. D. Radadia, Lab Chip 15, 4227-4231 (2015).
${ }^{36}$ C. O. Bounds, J. Upadhyay, N. Totaro, S. Thakuri, L. Garber, M. Vincent, Z. Huang, and J. A. Pojman, ACS Appl. Mater. Interfaces 5, 1643-1655 (2013).

${ }^{37}$ L. Garber, C. Chen, K. V. Kilchrist, C. Bounds, J. Pojman, and D. Hayes, J. Biomed. Mater. Res., Part A 101A, 3531-3541 (2013).

${ }^{38}$ C. Chen, L. Garber, M. Smoak, C. Fargason, T. Scherr, C. Blackburn, S. Bacchus, M. J. Lopez, J. A. Pojman, F. Del Piero, and D. J. Hayes, Tissue Eng., Part A 21, 320-331 (2015).

${ }^{39}$ C. D. Pritchard, T. M. O’Shea, D. J. Siegwart, E. Calo, D. G. Anderson, F. M. Reynolds, J. A. Thomas, J. R. Slotkin, E. J. Woodard, and R. Langer, Biomaterials 32(2), 587-597 (2011).

${ }^{40}$ E. Jee, T. Bánsági, A. F. Taylor, and J. A. Pojman, Angew. Chem., Int. Ed. 128(6), 2167-2171 (2016).

${ }^{41}$ A. Napoli, N. Tirelli, G. Kilcher, and A. Hubbell, Macromolecules 34(26), 8913-8917 (2001).

${ }^{42}$ J. Rieger, K. Van Butsele, P. Lecomte, C. Detrembleur, R. Jerome, and C. Jerome, Chem. Commun. 2005(2), 274-276.

${ }^{43}$ J. W. Chan, B. Yu, C. E. Hoyle, and A. B. Lowe, Chem. Commun. 2008(40), 4959-4961.

${ }^{44}$ S. Chatani, M. Podgórski, C. Wang, and C. N. Bowman, Macromolecules 47(15), 4894-4900 (2014).

${ }^{45}$ P. Bunton, D. Marin, E. Stewart, E. Meiburg, and A. De Wit, Exp. Fluids 57, 28 (2016).

${ }^{46}$ F. Haudin, L. A. Riolfo, B. Knaepen, G. M. Homsy, and A. De Wit, Phys. Fluids 26, 044102 (2014).

${ }^{47}$ D. Schafroth, N. Goyal, and E. Meiburg, Eur. J. Mech. - B/Fluids 26(3), 444-453 (2007).

${ }^{48}$ S. H. Hejazi, P. M. J. Trevelyan, J. Azaiez, and A. De Wit, J. Fluid Mech. 652, 501-528 (2010).

${ }^{49}$ See http://www.glossary.oilfield.slb.com/Terms/s/secondary_recovery.aspx for Schlumberger. 